\title{
Chromothripsis after Stumbling through DNA Replication
}

Most structural genome variations (SVs), such as chromosome translocations and inversions, are readily detected by classical chromosome banding in combination with molecular cytogenetic techniques. With steadily improving levels of resolution, the frequency of detection of SVs increases. At nucleotide resolution, these techniques will also allow us to investigate the sequences of the break points of the SVs and eventually their possible mechanism of origin. Using FISH, array CGH, Southern blot hybridization and MLPA, the break points of SVs were mapped, which then allowed us to determine the sequences surrounding the fusion points of these breaks [Higgins et al., 2008; Sobreira et al., 2011; Chiang et al., 2012]. Only one out of 17 fusions was perfectly balanced at the nucleiotide level [Higgins et al., 2008]. The overwhelming majority, roughly three quarters, involved insertions, deletions and stretches of microhomology of a few base pairs (bp), which is consistent with nonhomologous end joining (NHEJ). The remainder may have resulted from microhomology-mediated break-induced replication (MMBIR), since they involved more extensive DNA synthesis [Higgins et al., 2008; Sobreira et al., 2011; Chiang et al., 2012]. In a study of 8 de novo unbalanced translocations, which most likely occurred postzygotically, 5 indicated nonallelic homologous recombination between paralogous repeats as the predominant mechanism [Robberecht et al., 2013]. Apparently, at least 3 distinct pathways of 'repair' of chromosome breaks exist.
Analysis of 51 reciprocal translocations and $6 \mathrm{SV}$ s with more than 3 break points involving 2-5 chromosomes showed even more complex patterns [Weckselblatt et al., 2015]. Sequencing 37 of the fusion points of reciprocal translocations revealed microhomologies between 0 and 4 bp $(n=26)$, short inserted sequences $(n=8)$, or paralogous repeats $(\mathrm{n}=3)$, consistent with NHEJ and MMBIR. In 3 reciprocal translocations, fusion genes were predicted, which may lead to gain of function, but this was not confirmed by mRNA sequencing. In 2 out of 3 cases of complex translocations with associated inversions and insertions, 18 and 33 fusions involving up to 5 different chromosomes were found. The large number of break points and their relatively close spacing suggests that these SVs arose after chromothripsis. All fusion points had $0-4$ bp of microhomology, consistent with NHEJ, and both SV s occurred as de novo events on paternal alleles. Together with other studies, these data suggest that germline chromothripsis arises in the paternal genome and may be transmitted maternally [Kloosterman et al., 2011, 2012; Chiang et al., 2012; Nazaryan et al., 2014; Pellestor et al., 2014; Poot and Haaf, 2015; Weckselblatt et al., 2015].

While all of these studies indicated possible mechanisms of fusions, they did not reveal the mechansim(s) of breakage that produced the SV. Thus far, mechanisms such as excessive levels of double-strand breaks (DSBs) during meiosis, mismatching DSB repair during DNA

\section{KARGER 125}

(c) 2015 S. Karger AG, Base

1661-8769/15/0065-0207\$39.50/0
Martin Poot

Department of Human Genetics, University of Würzburg

Biozentrum, Am Hubland

DE-97074 Würzburg (Germany)

E-Mail Martin_Poot@hotmail.com 
replication or during meiosis, or postzygotic rearrangements have been suggested [Hart and O'Driscoll, 2013; Robberecht et al., 2013; Pellestor et al., 2014; Poot and Haaf, 2015]. The most extreme form of SV is germline chromothripsis, i.e. shattering of one or a few chromosomes and stitching together the resulting fragments in random order and orientation [Kloosterman and Hochstenbach, 2014; Pellestor et al., 2014; Poot and Haaf, 2015]. The chromosome shattering step of chromothripsis is poorly understood, but in a recent study, chromothripsis has been 'caught in the act' [Zhang et al., 2015].

After release of retinal pigment epithelial cells in culture from nocodazole arrest, micronulcei, containing one or several chromosomes, were formed [Zhang et al., 2015]. Upon entry into the $S$ phase of the cell cycle, as demonstrated by 5 -ethynyl-2' -deoxyuridine (EdU) labeling, these underwent DNA damage as shown by fluorescence labeling for the phosphorylated form of histone H2AX. Since their level of EdU labeling was lower than that of the mother nucleus, replication of the chromosomes in the micronuclei was incomplete. After completion of one cell cycle, the EdU-positive micronuclei reincorporated into the mother nucleus. These cells were then isolated and their genomes sequenced. Some cells lost and some cells gained one or several chromosomes. To determine what has happened to the chromosomes in the micronuclei, the authors studied nuclei which had gained a chromosome or part of it. Nuclei with chromosome gains showed 12.5-fold higher rates of SVs. Thus, rearrangements $>150 \mathrm{~kb}$ and interchromosomal rearrangements, such as insertion translocations, and inversions within $150 \mathrm{~kb}$ from a break point were significantly more frequent in nuclei with gains, which is in agreement with chromothripsis only. By SNP haplotyping, the authors found that all rearrangements had exclusively taken place on the missegregated and reincorporated chromosome. More than $50 \%$ of the fusion points of the rearrangements showed extensive microhomologies and contained insertions ranging from 50 to $500 \mathrm{bp}$, which is consistent with MMBIR as the fusion mechansism. This mechanism, which requires one round of DNA replication, is in agreement with several, but not all of the thus far described cases of germline chromothripsis [Kloosterman et al., 2011, 2012; Nazaryan et al., 2014].

These observations also bear on chromosomal instability disorders, such as Werner syndrome (WRN) and Bloom syndrome (BLM). WRN is associated with a variegated translocation mosaicism, a prolongation of the $S$ phase of the cell cycle, hypersensitivity to DNA cross-linking and DSBs provoking agents, and a reduction in recombina- tional DSB repair [Salk et al., 1981; Poot et al., 1992, 2001, 2002; Ogburn et al., 1997; Melcher et al., 2000; Dhillon et al., 2007]. In BLM, rates of sister chromatid exchanges and hypersensitivity to DSB-causing agents are elevated [Bischof et al., 2001]. Cultured lymphoblastic cells from both WRN and BLM patients show spontaneously elevated levels of micronuclei, which further increased after treatment with compounds that interfered with DNA replication [Honma et al., 2002]. Most micronuclei accumulate DSBs, which contain phosphorylated H2AX and the RAD51 protein [Haaf et al., 1999; Crasta et al., 2012]. The latter is mobilized by the BLM protein to sites of DSBs [Wu and Hickson, 2003; Haber, 2015]. Thus, these observations link the chromosomal instability of WRN and BLM to this novel micronuclei-related form of chromothripsis.

In families in which SVs have resulted from germline chromothripsis, this appeared to be an isolated event [Poot et al., 2009; Kloosterman et al., 2011, 2012]. In somatic cells, chromothripsis is particularly frequent in malignancies of mesenchymal tissues and increases with the age of the patient [Cai et al., 2014]. A strongly increased risk of malignancies of mesenchymal tissues, likely due to the continuous genomic instability of somatic cells, is also a hallmark of WRN [Salk et al., 1981; Poot et al., 1992, 2001, 2002; Ogburn et al., 1997; Melcher et al., 2000; Honma et al., 2002; Dhillon et al., 2007]. While lymphoid cells from WRN patients respond to DNA damage with apoptosis, mesenchymal cells show growth arrest leading to a state of cellular senescence [Poot et al., 2002]. The latter ties in with the premature ageing of WRN patients and suggests that DNA damage-induced perturbation of DNA replication, somatic cell chromothrispsis, risk of malignant disease of mesenchymal tissues, and ageing are somehow interconnected.

While in the study of Zhang et al. [2015], cultured epithelial cells, which were made to 'stumble' at the G1/S border of the cell cycle by nocodazole treatment, gave us a novel insight into how chromothripsis may arise, it also prompted numerous novel questions. Some cases of chromothripsis show evidence of MMBIR, in agreement with micronucleus-dependent chromosome shattering, while others are consistent with NHEJ. This suggests that at least one other mechanism of chromosome shattering may exist. In addition, the here described studies indicate that several methods of structural genome analysis have to be applied concomitantly to grasp the full complexity of SV s in general and chromothripsis in particular [Kloosterman et al., 2011, 2012; Nazaryan et al., 2014; Weckselblatt et al., 2015].

Martin Poot 


\section{References}

-Bischof O, Kim SH, Irving J, Beresten S, Ellis NA, Campisi J: Regulation and localization of the Bloom syndrome protein in response to DNA damage. J Cell Biol 153:367-380 (2001).

-Cai H, Kumar N, Bagheri HC, von Mering C, Robinson MD, Baudis M: Chromothripsislike patterns are recurring but heterogeneously distributed features in a survey of 22,347 cancer genome screens. BMC Genomics 15: 82 (2014).

Chiang C, Jacobsen JC, Ernst C, Hanscom C, Heilbut A, et al: Complex reorganization and predominant non-homologous repair following chromosomal breakage in karyotypically balanced germline rearrangements and transgenic integration. Nat Genet 44:390-397 (2012).

-Crasta K, Ganem NJ, Dagher R, Lantermann AB, Ivanova $\mathrm{EV}$, et al: DNA breaks and chromosome pulverization from errors in mitosis. Nature 482:53-58 (2012).

- Dhillon KK, Sidorova J, Saintigny Y, Poot M, Gollahon K, et al: Functional role of the Werner syndrome RecQ helicase in human fibroblasts. Aging Cell 6:53-61 (2007).

Haaf T, Raderschall E, Reddy G, Ward DC, Radding CM, Golub EI: Sequestration of mammalian Rad51-recombination protein into micronuclei. J Cell Biol 144:11-20 (1999).

Haber JE: TOPping Off Meiosis. Mol Cell 57:577581 (2015).

Hart L, O'Driscoll M: Causes and consequences of structural genomic alterations in the human genome. eLS 2013, DOI: 10.1002/ 9780470015902.a0024976.

- Higgins AW, Alkuraya FS, Bosco AF, Brown KK, Bruns GA, et al: Characterization of apparently balanced chromosomal rearrangements from the developmental genome anatomy project. Am J Hum Genet 82:712-722 (2008).

-Honma M, Tadokoro S, Sakamoto H, Tanabe H, Sugimoto M, et al: Chromosomal instability in B-lymphoblasotoid cell lines from Werner and Bloom syndrome patients. Mutat Res 520:15-24 (2002).

Kloosterman WP, Hochstenbach R: Deciphering the pathogenic consequences of chromosomal aberrations in human genetic disease. $\mathrm{Mol}$ Cytogenet 7:100 (2014).

Kloosterman WP, Guryev V, van Roosmalen M, Duran KJ, de Bruijn E, et al: Chromothripsis as a mechanism driving complex de novo structural rearrangements in the germline. Hum Mol Genet 20:1916-1924 (2011).

Kloosterman WP, Tavakoli-Yaraki M, van Roosmalen MJ, van Binsbergen E, Renkens I, et al: Constitutional chromothripsis rearrangements involve clustered double-stranded DNA breaks and nonhomologous repair mechanisms. Cell Rep 1:648-655 (2012).

- Melcher R, von Golitschek R, Steinlein C, Schindler D, Neitzel H, et al: Spectral karyotyping of Werner syndrome fibroblast cultures. Cytogenet Cell Genet 91:180-185 (2000).

Nazaryan L, Stefanou EG, Hansen C, Kosyakova $\mathrm{N}$, Bak M, et al: The strength of combined cytogenetic and mate-pair sequencing techniques illustrated by a germline chromothripsis rearrangement involving FOXP2. Eur J Hum Genet 22:338-343 (2014).

- Ogburn CE, Oshima J, Poot M, Chen R, Hunt KE, et al: An apoptosis-inducing genotoxin differentiates heterozygotic carriers for Werner helicase mutations from wild-type and homozygous mutants. Hum Genet 101:121-125 (1997).

Pellestor F, Gatinois V, Puechberty J, Geneviève D, Lefort G: Chromothripsis: potential origin in gametogenesis and preimplantation cell divisions. A review. Fertil Steril 102:1785-1796 (2014).

Poot M, Haaf T: Mechanisms of origin, phenotypic effects and diagnostic implications of complex chromosome rearrangements. Mol Syndromol 6:109-133 (2015).

Poot M, Hoehn H, Rünger TM, Martin GM: Impaired S-phase transit of Werner syndrome cells expressed in lymphoblastoid cell lines. Exp Cell Res 202:267-273 (1992).

Poot M, Yom JS, Whang SH, Kato JT, Gollahon KA, Rabinovitch PS: Werner syndrome cells are sensitive to DNA cross-linking drugs. FASEB J 15:1224-1226 (2001).

Poot M, Gollahon KA, Emond MJ, Silber JR, Rabinovitch PS: Werner syndrome diploid fibroblasts are sensitive to 4-nitroquinoline- $\mathrm{N}$ oxide and 8-methoxypsoralen: implications for the disease phenotype. FASEB J 16:757758 (2002).

Robberecht C, Voet T, Zamani Esteki M, Nowakowska BA, Vermeesch JR: Nonallelic homologous recombination between retrotransposable elements is a driver of de novo unbalanced translocations. Genome Res 23: 411-418 (2013).

Salk D, Au K, Hoehn H, Martin GM: Cytogenetics of Werner's syndrome cultured skin fibroblasts: variegated translocation mosaicism. Cytogenet Cell Genet 30:92-107 (1981).

Sobreira NL, Gnanakkan V, Walsh M, Marosy B, Wohler E, et al: Characterization of complex chromosomal rearrangements by targeted capture and next-generation sequencing. Genome Res 21:1720-1727 (2011).

-Weckselblatt B, Hermetz KE, Rudd MK: Unbalanced translocations arise from diverse mutational mechanisms including chromothripsis. Genome Res 25:937-947 (2015).

Wu L, Hickson ID: The Bloom's syndrome helicase suppresses crossing over during homologous recombination. Nature 426:870-874 (2003).

Z Zhang CZ, Spektor A, Cornils H, Francis JM, Jackson EK, et al: Chromothripsis from DNA damage in micronuclei. Nature 522:179-184 (2015). 